\title{
Regeneration of Lesioned Corticospinal Tract Fibers in the Adult Rat Induced by a Recombinant, Humanized IN-1 Antibody Fragment
}

\author{
Christian Brösamle, ${ }^{1}$ Andrea B. Huber, ${ }^{1}$ Markus Fiedler, ${ }^{2}$ Arne Skerra, ${ }^{2}$ and Martin E. Schwab ${ }^{1}$ \\ ${ }_{1}^{1}$ Brain Research Institute, Department of Neuromorphology, University of Zurich and Swiss Federal Institute of Technology, \\ 9057 Zurich, Switzerland, and 2Lehrstuhl für Biologische Chemie, Technische Universität München, D-85350 \\ Freising-Weihenstephan, Germany
}

\begin{abstract}
Axons in the CNS of higher vertebrates generally fail to regenerate after injury. This lack of regeneration is crucially influenced by neurite growth inhibitory protein constituents of CNS myelin. We have shown previously that a monoclonal antibody (mAb IN-1) capable of binding and neutralizing Nogo-A, a myelin-associated inhibitor of neurite growth, can induce long-distance axonal regeneration and increased structural plasticity with improved functional recovery in rat models of CNS injury. In this paper we demonstrate that a partially humanized, recombinant Fab fragment (rIN-1 Fab) derived from the original mAb IN-1, was able to promote long-distance regeneration of injured axons in the spinal cord of adult rats. When infused into a spinal cord injury site, regrowth of corticospinal fibers in 11 of 18 animals was observed after a survival time of 2 weeks. Regenerating fibers grew for $>9$
\end{abstract}

$\mathrm{mm}$ beyond the lesion site and arborized profusely in the distal cord. Regenerated fibers formed terminal arbors with varicosities in the spinal cord gray matter, strongly resembling synaptic points of contact to neurons in the spinal cord distal to the lesion. In animals that had received a bovine serum albumin solution or a recombinant $\mathrm{IN}-1$ fragment that had been mutated in the antigen binding site (mutIN-1 Fab), no significant growth beyond normal lesion-induced sprouting was observed. Neutralization of endogenous nerve growth inhibitors represents a novel use of recombinant antibody technology with potential therapeutic applications after traumatic CNS lesions.

Key words: CNS regeneration; Nogo- $A$; spinal cord injury; axonal tracing; recombinant antibody; corticospinal tract; rat
Injury to the adult CNS of humans or higher vertebrates results in permanent structural damage that in turn leads to no or only incomplete functional recovery (Ramon y Cajal, 1928; Schwab and Bartholdi, 1996). CNS myelin constituents that are strongly inhibitory to neurite growth have been shown to contribute to this failure of regeneration (Schwab and Thoenen, 1985; Schwab and Caroni, 1988; Schwab et al., 1993). We have purified and characterized one of these inhibitors, Nogo-A (Schwab and Caroni, 1988; Spillmann et al., 1998; Chen et al., 2000), and its cDNA has been cloned recently (Chen et al., 2000; GrandPré et al., 2000). A monoclonal antibody (mAb IN-1) was raised against a myelin fraction enriched in Nogo-A that was able to bind Nogo-A and neutralize its neurite growth inhibitory properties. This mAb IN-1 was shown to be able to promote neurite extension on inhibitory myelin substrates in culture (Caroni and Schwab, 1988; Rubin et al., 1994). When applied to various CNS lesions in an adult rat, $\mathrm{mAb}$ IN-1 can induce regenerative long-distance fiber growth (Schnell and Schwab, 1990, 1993; Cadelli and Schwab, 1991; Schnell et al., 1994; Weibel et al., 1994). Recently, we showed that application of mAb IN-1 can also enhance compensatory sprouting of intact nerve fiber systems in response to a lesion (Thallmair et al., 1998; Z'Graggen et al., 1998). The reestablishment of functional contacts was demonstrated by clear behavioral recovery in animals that were treated with $\mathrm{mAb}$ IN-1 in comparison to control rats (Bregman et al., 1995; Thallmair et al., 1998; Z'Graggen et al., 1998).

There are several problems attached to these experiments, how-

\footnotetext{
Received April 13, 2000; revised July 28, 2000; accepted Aug. 8, 2000.

This work was supported by the Swiss National Science Foundation (Grants 3145549.95 and 4038-43918), the Research Consortium on Spinal Cord Injury of the Christopher Reeve Paralysis Foundation, the International Research Institute for Paraplegiology (IFP, Zurich), private donations, and the Deutsche Forschungsgemeinschaft (Grant Sk 33/2-1). We thank Dr. Marjan van der Haar for recombinant Nogo-A and Tiziana Flego for technical assistance.

Correspondence should be addressed to Christian Brösamle, Brain Research Institute, University of Zurich, Winterthurer Strasse 190, 8057 Zürich, Switzerland. E-mail: broesam@hifo.unizh.ch.

Copyright (C) 2000 Society for Neuroscience $0270-6474 / 00 / 208061-08 \$ 15.00 / 0$
}

ever, mostly related to the fact that mAb IN-1, as an antibody of the immunoglobulin $(\mathrm{Ig}) \mathrm{M} / \kappa$ subclass, has relatively low stability when concentrated and stored. The antibody was typically administered to the CNS by implanting antibody-producing hybridoma cells into the brain, either encapsulated or directly as suspension, leading to tumor growth and immunological problems. An additional problem was the poorly controlled production and secretion of antibody.

Recently, it became possible to clone the DNA sequences coding for antibody chains and to express tailor-made recombinant antibodies in large quantities in bacteria (Skerra and Plückthun, 1988; Orlandi et al., 1989). We have cloned the cDNAs for the variable regions of the Fab light and heavy chain, including the antigen binding site of mAb IN-1, and expressed a monovalent, recombinant fragment, rIN-1 Fab ( $\sim 45 \mathrm{kDa}$ molecular mass $)$, that was able to neutralize Nogo-A in vitro (Bandtlow et al., 1996). rIN-1 Fab can be produced in large quantities, concentrated, stored, and infused as a pure reagent to the CNS in a way potentially also applicable to human patients. To this end we now have replaced the murine constant domains with human domains that should make the molecule more acceptable to the human immune system. In this paper we report the regeneration-inducing effect of the recombinant, humanized IN-1 Fab fragment in a rat model of spinal injury. In addition, we show the exact course and the terminations of regenerated axons in the spinal cord caudal to the lesion.

\section{MATERIALS AND METHODS}

Bacterial production and purification of rIN-1 Fab. Using the previously described vector pASK85-IN1 for the expression of the murine rIN-1 Fab fragment (Bandtlow et al., 1996), the coding regions for the constant domains were replaced by cloned cDNAs for the corresponding human Ig domains using standardized restriction sites (Schiweck et al., 1997). In addition, the $\beta$-lactamase-encoding gene was either precisely substituted by the chloramphenicol $(\mathrm{Cm})$ acetyl transferase gene, yielding pASK116-IN1, or the $\mathrm{Cm}^{\mathrm{r}}$ gene was inserted as a second resistance marker upstream of the tet promoter region (M. Fiedler and A. Skerra, unpublished). Laboratory fermentation was essentially performed as described (Schiweck and Skerra, 1995) with Escherichia coli K12 strain W3110 in a synthetic medium, and final cell densities of $\mathrm{OD}_{550}=40$ on average were reached. The rIN-1 Fab fragment was liberated from the bacterial periplasm by EDTA/ lysozyme treatment and purified to apparent homogeneity by means of its 
A

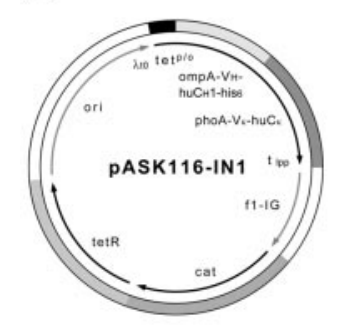

B

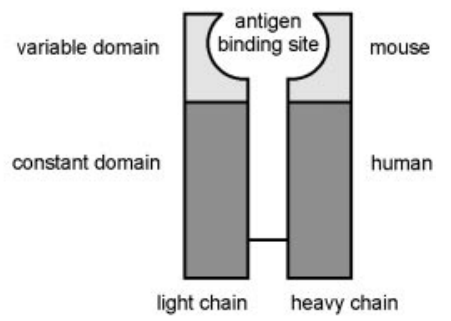

Figure 1. Recombinant, humanized IN-1 Fab. $A$, Expression vector pASK116-IN1. The two chains of the Fab fragment are fused with the bacterial OmpA and PhoA signal sequences, respectively, and the heavy chain carries a $\mathrm{His}_{6}$ tag at its $\mathrm{C}$ terminus. The structural genes for both polypeptide chains are arranged in a dicistronic operon under transcriptional control of the tet promoter/operator $\left(t e t^{p / o}\right)$, ending with the lipoprotein terminator $\left(t_{l p p}\right)$. Tight repression of the tet promoter is controlled by the tet repressor gene $(t e t R)$. ori, Origin of replication; $\lambda_{t o}$, phage $\lambda$ transcription terminator; $f 1-I G$, intergenic region of filamentous phage $\mathrm{f} 1$; cat, $\mathrm{Cm}$ resistance gene. $B$, The recombinant IN-1 Fab carries the variable domains of the original (murine) IgM/ $/ \kappa \mathrm{mAb}$ IN-1 determining the antigen specificity and constant domains of human origin, $\mathrm{IgG} 1 / \kappa$ subclass.

$\mathrm{His}_{6}$ tag in two rounds of immobilized metal affinity chromatography. The protein solution was finally dialyzed against cerebrospinal buffer (CSB) composed of (in mM): $110 \mathrm{NaCl}, 1.5 \mathrm{CaCl}_{2}, 1.5 \mathrm{MgCl}_{2}, 4 \mathrm{KCl}, 30$ $\mathrm{NaHCO}_{3}, 1$ citric acid, $\mathrm{pH}$ 7.2, concentrated on an Amicon YM10 membrane to $5 \mathrm{mg} / \mathrm{ml}$, and sterilized by filtration. A mutated rIN-1 Fab (mut rIN-1 Fab) was obtained by site-directed mutagenesis of an alanine residue to phenylalanine at position 32 of the VL domain located within the antigen binding site (Fiedler and Skerra, 1999). In vitro assays (see Fig. 3) showed that this mutation completely abolished the Nogo-A-neutralizing properties, and this mutated Fab was therefore used as a control in some of the in vivo experiments. To assess the distribution of rIN-1 Fab delivered to the spinal cord, rIN-1 Fab was labeled by the fluorescent dye Alexa488 (Molecular Probes) according to the manufacturer's instructions. Labeled rIN-1 Fab was prepared at a concentration of $0.77 \mathrm{mg} / \mathrm{ml}$, carrying 3.3 dye molecules per molecule, and was used at that concentration for inf usion into animals $(n=3)$ as described below.

Immunohistochemistry and Western blotting. Rats were decapitated, and fresh spinal cord tissue was embedded and frozen at $-40^{\circ} \mathrm{C}$ in OCT compound (Tissuetek, Zoeterwoude, The Netherlands). Cryosections (20 $\mu \mathrm{m})$ were taken on coated slides and stained essentially as described (Rubin et al., 1994). Recombinant humanized IN-1 Fab was used at a concentration of $50 \mu \mathrm{l} / \mathrm{ml}$ and detected by a secondary horseradish peroxidase (HRP)-coupled antibody directed against the human $\kappa$ light chain of the humanized part of the rIN-1 Fab (1:100, Dako, Copenhagen, Denmark). For Western blotting, recombinant myc-tagged, full-length Nogo-A, previously shown to exhibit strong neurite growth-inhibiting activity (Chen et al., 2000) $5 \mu \mathrm{g}$ whole Chinese hamster ovary $(\mathrm{CHO})$ cell lysate per lane] was run on SDS-PAGE and blotted onto a polyvinylidene difluoride membrane (Millipore, Bedford, MA). Blot stripes were incubated with $\mathrm{rIN}-1$ Fab $(10 \mu \mathrm{g} / \mathrm{ml})$ and a secondary antibody against the human $\kappa$ light chain coupled to HRP (1:100, Dako). Stripes were stripped and reincubated in anti-myc mAb (1:5000) followed by a rabbit anti-mouseHRP secondary antibody (1:30,000, both from Invitrogen, San Diego, CA) to reveal myc-tagged recombinant protein. HRP activities were visualized using a chemiluminescence system (SuperSignal, Pierce, Rockford, IL).

In vitro bioassays. All bioassays were scored by a person blind to the experimental conditions.

The 3T3 spreading assay was performed as described (Spillmann et al., 1997). Partially purified inhibitory activity of bovine spinal cord (q-pool) (Rubin et al., 1995) was used for coating four-well culture dishes $(5 \mu \mathrm{g} / 1$ $\mathrm{cm}^{2}$ well; Greiner) overnight at $4^{\circ} \mathrm{C}$. Eight thousand 3T3 fibroblasts in 100 $\mu \mathrm{l}$ DMEM with $10 \%$ fetal calf serum (FCS) were plated on each well. After $30 \mathrm{~min}$ incubation at $37^{\circ} \mathrm{C}$, the assay was stopped by adding $4 \%$ formaldehyde in $0.1 \mathrm{~m}$ phosphate buffer, $\mathrm{pH} 7.0$, with $5 \%$ sucrose. To compensate for activity variations in different q-pool preparations, the number of inhibited, round cells plated on q-pool $(80-90 \%$ of total cells $)$ was normalized to $100 \%$ and to $0 \%$ for those plated on a buffer control (10-25\% of total cells).

For treatment with antibodies, the coated dishes were preincubated for $15 \mathrm{~min}$ at $37^{\circ} \mathrm{C}$ with undiluted $\mathrm{mAb} \mathrm{IN}-1$ hybridoma supernatant $(100$ $\mu \mathrm{l} /$ well; $1-10 \mu \mathrm{g}$ antibody/ml), with rIN-1 Fab, or with mut rIN-1 Fab at concentrations of 500,50 , and $5 \mu \mathrm{g} / \mathrm{ml}$ in PBS before plating of the 3T3 cells.

Dorsal root ganglia (DRGs) were dissected from embryonic day 16 (E16) chicken in HBSS and plated on dishes precoated with q-pool (as described for the 3T3 assay) in $100 \mu \mathrm{l} \mathrm{F12} \mathrm{medium} \mathrm{with} 10 \%$ FCS and $1 \%$ methyl-cellulose. Each explant (DRG) was divided into two parts. Neurite outgrowth from individual DRGs was scored after $24 \mathrm{hr}$ incubation at $37^{\circ} \mathrm{C}$

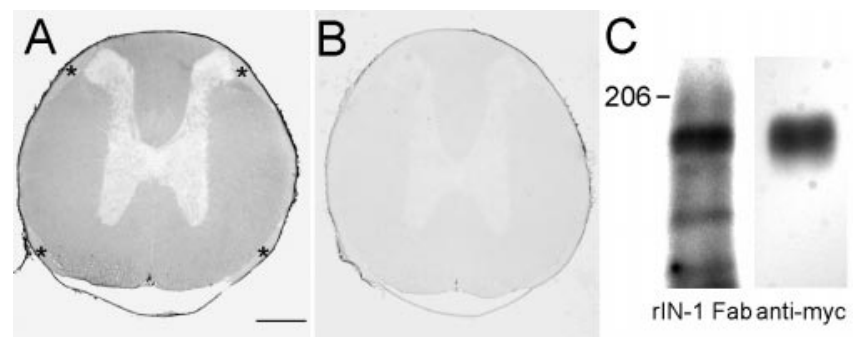

Figure 2. rIN-1 Fab stains CNS white matter and recognizes recombinant Nogo-A. $A$, Recombinant, humanized IN-1 Fab stains myelinated structures in the CNS. Unmyelinated areas such as the superficial laminae of the spinal cord and peripheral myelinated tissues (dorsal and ventral roots, asterisks) are not or only weakly stained. This staining pattern very closely resembles that of the original IN- $1 \mathrm{mAb}$. $B$, Sections incubated with the secondary antibody only were not significantly stained. Scale bar, $300 \mu \mathrm{m}$. $C$, Recombinant, full-length Nogo-A prepared from stably transfected $\mathrm{CHO}$ cells is recognized by rIN-1 Fab on Western blot as shown by reprobing the same blot with an anti-myc antibody to reveal recombinant protein.

in a semiquantitative way using a scale of 0 (no outgrowth) to 4 (maximum outgrowth).

Surgery. All surgical procedures were approved by the authorities of the canton of Zurich. Female Lewis rats, 6-8 weeks of age, from our own breeding colony, were deeply anesthetized with fentanyl citrate $(0.0189$ $\mathrm{mg} / 100 \mathrm{gm})$, fluanisone $(0.6 \mathrm{mg} / 100 \mathrm{gm}$, Hypnorm, Janssen $)$, and midazolam (0.6 mg/100 gm, Dormicum, Roche). Laminectomies were performed at spinal levels T8 and T10, and the spinal cord was exposed. With fine iridectomy scissors, a dorsolateral hemisection was performed at level T8, completely interrupting the main dorsomedial and the minor dorsolateral corticospinal tract (CST) components. An Alzet 2002 osmotic mini-pump $(\sim 240 \mu \mathrm{l}$ fill volume, $0.5 \mu \mathrm{l} / \mathrm{hr}$ mean pumping rate, $14 \mathrm{~d}$ delivery) filled with either rIN-1 Fab, mut rIN-1 Fab, or bovine serum albumin (BSA) as a control (always at $5 \mathrm{mg} / \mathrm{ml}$ in CSB), was placed under the skin on the back of the animal. The catheter connected to the outlet of the pump was inserted at level T10 from caudal through a small hole in the dura into the intrathecal space onto the spinal cord close to the lesion (see Fig. 4) and fixed in place by suturing to the surrounding tissue. The muscle layers over the laminectomies were sutured, and the skin on the back was closed with surgical clamps. The scalp of the head was cut, and on both sides a hole was drilled into the skull overlying the sensorimotor cortex. By three shallow injections, $2.5 \mu$ l of the anterograde neuronal tracer biotin dextrane amine (BDA; $10 \%$ in PB, Molecular Probes) was applied. The scalp was sutured, and the animals were left to recover on a heat pad.

Histology and analysis. After a survival time of 2 weeks, the animals were killed by a pentobarbital overdose $(50 \mathrm{mg} / 100 \mathrm{gm})$ and perfused transcardially with a Ringer's solution containing 100,000 I U heparin and $0.25 \%$ $\mathrm{NaNO}_{2}$, followed by a $4 \%$ formaldehyde solution in $0.1 \mathrm{M}$ phosphate buffer $+5 \%$ sucrose. The spinal cords were dissected from the animals, post-fixed overnight in the same fixative, and embedded in a gelatin-albumin matrix polymerized by glutaraldehyde (Herzog and Brösamle, 1997). Complete series of consecutive sagittal sections (50 $\mu \mathrm{m}$ thick) were taken on a vibratome and processed with avidin-HRP (ABC elite, Vector Laboratories, Burlingame, CA) followed by a nickel-enhanced diaminobenzidine HRP reaction for the visualization of the BDA tracer, in a semifreefloating technique as described earlier (Herzog and Brösamle, 1997). The microscopic slides were analyzed on an Olympus microscope and drawings were made at a magnification of $50 \times$ with a camera lucida tubus attached to the microscope. Only fibers of the main and the lateral CST components were drawn, and fibers of the small ventral CST component (Brösamle and Schwab, 1997) were omitted. Animals with incomplete lesions, identified by the straight trajectory of the unlesioned fibers through the lesion and further on throughout the whole tissue block, or lesions that did not leave a ventral tissue bridge (6 of 31) were excluded from the analysis. Regenerative fiber growth was measured from the center of the lesion to the caudalmost extend of CST fibers that could be traced back to the lesioned dorsomedial CST. Fiber growth from the lesioned CST stump beyond the center of the lesion was scored as "long-distance regeneration." Photomicrographs were taken through a JVC high-resolution CCD camera and Image Access software (Imagic, Glattbrugg, Switzerland). Fluorescence micrographs were taken on a Zeiss Axiophot through a Xillix slow-scan CCD camera using MCID software. Images were assembled in Photoshop (Adobe), and contrast was adjusted and sharpened when necessary.

\section{RESULTS}

\section{Bacterial expression and purification of humanized rIN-1 Fab}

A functional rIN-1 Fab fragment was produced in E. coli by coexpressing its light and heavy chains, each fused with a bacterial 
A

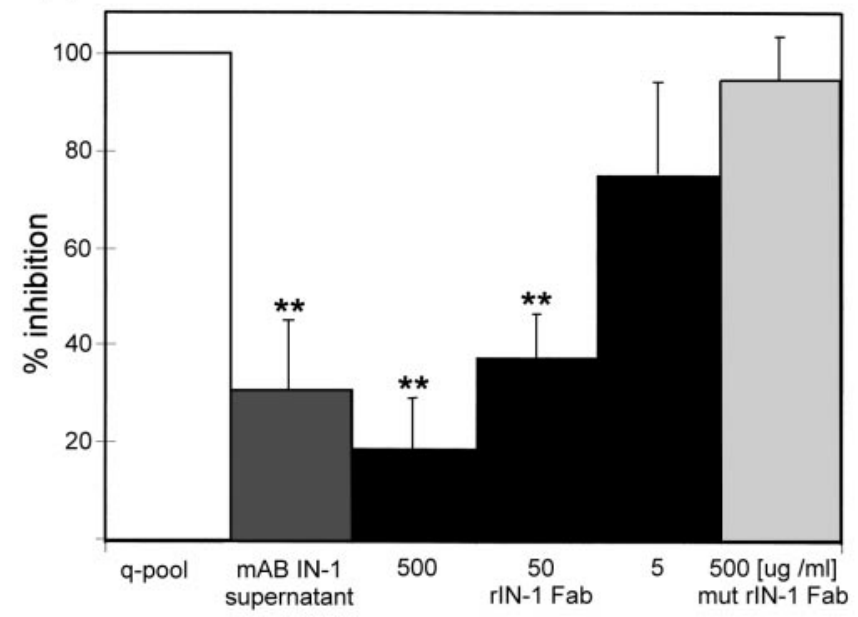

B

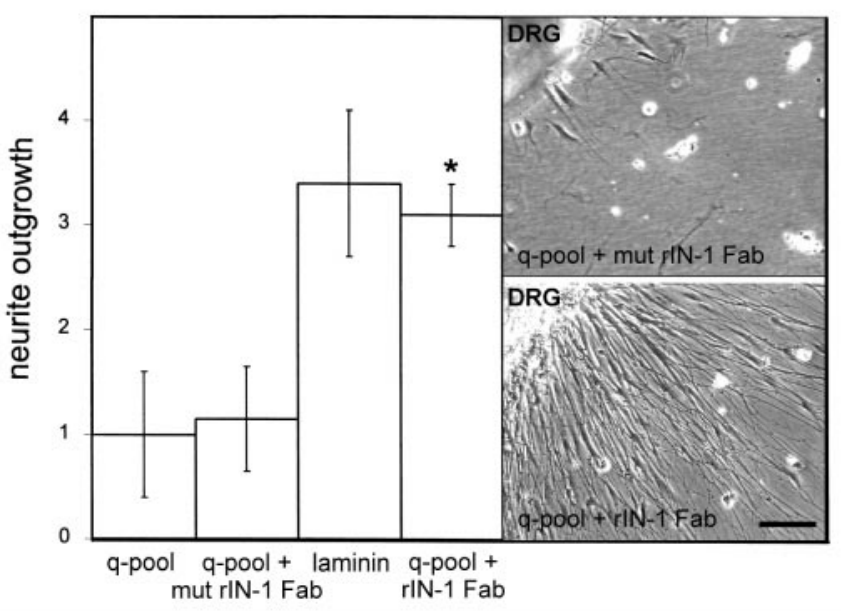

A

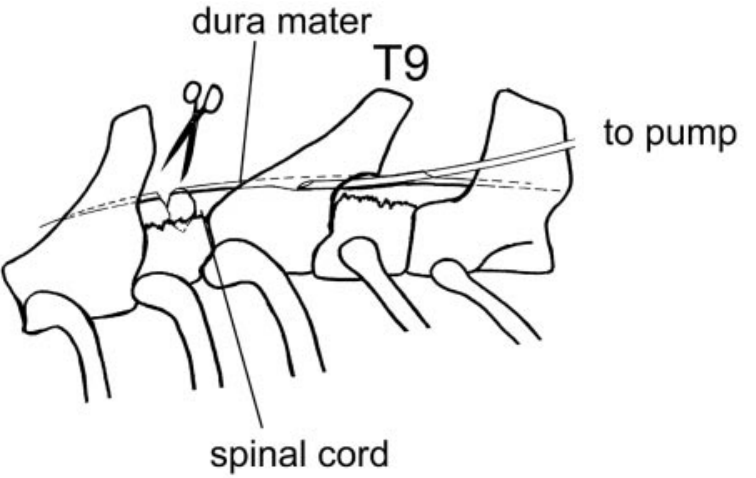

Figure 4. Spinal cord injury model and subdural antibody infusion. $A$, Laminectomies were performed at levels T8 and T10. With fine iridectomy scissors, the dorsal half of the spinal cord was cut bilaterally at T8, thus transecting the main dorsomedial and the minor dorsolateral corticospinal tract components. Through a small hole in the dura a catheter connected to an osmotic mini-pump was inserted into the subdural space close to the lesion at T8 to infuse the lesion area and the distal cord with rIN-1 Fab solution. $B$, Distribution of inf used rIN-1 Fab was controlled by labeling the antibodies with the fluorochrome Alexa488. After 1 week of inf usion, rIN-1 Fab had spread well at the infusion site, around the lesion (asterisk), and in the distal cord. rIN-1 Fab concentration was higher in gray matter, but Alexa488 fluorescence could also be easily detected in white matter. Macrophages that had infiltrated the lesion (arrowheads) exhibited strong bluegreen fluorescence caused by uptake of the infused rIN-1 Fab and yellowish-green autofluorescence. Scale bar, $500 \mu \mathrm{m}$.

Figure 3. Humanized rIN-1 Fab recognizes and neutralizes myelinassociated neurite growth inhibitors in vitro. $A, 3 \mathrm{~T} 3$ fibroblast spreading is inhibited when the cells are plated on a CNS myelin protein substrate enriched for Nogo-A (q-pool) (Spillmann et al., 1997, 1998). When the inhibitory substrate is pretreated with mAb IN-1 hybridoma supernatant $(50 \mu \mathrm{g} / \mathrm{ml}$ IgM), inhibition is reduced drastically. rIN-1 Fab has a similar neutralizing effect at concentrations of $500-50 \mu \mathrm{g} / \mathrm{ml}$ and is less efficient at higher dilutions. Mutated rIN-1 Fab did not show neutralizing activity. $* * p<0.01$, Student's $t$ test, compared with q-pool $(100 \%)$. B, Neurite outgrowth from E16 chick dorsal root ganglia is strongly inhibited when the DRGs are plated on q-pool. Pretreatment with humanized rIN-1 Fab allows for outgrowth of large numbers of long neurites within $24 \mathrm{hr}$. Mutated rIN-1 Fab did not neutralize Nogo-A in the q-pool substrate. Neurite outgrowth was scored in arbitrary units from 0 (no outgrowth) to 4 (maximal outgrowth on a laminin substrate). ${ }^{*} p<0.05$, Student's $t$ test, compared with q-pool. Scale bar, $30 \mu \mathrm{m}$. Error bars indicate means \pm SEM.

signal peptide. Thus the two polypeptides were secreted into the periplasmic space, where the formation of the disulfide bonds (one in each of the four Ig domains plus a fifth one between the two chains) and the folding and chain association of the antibody fragment took place (Plückthun and Skerra, 1989). The rIN-1 Fab was produced in a bench-top fermenter at high bacterial cell density using the tightly controlled tet ${ }^{\mathrm{p} / \mathrm{o}}$ system (Schiweck and Skerra, 1995). For the humanization of the murine antibody fragment, the cDNAs for the two variable domains derived from the IN-1 IgM/ $/ \kappa$ antibody were fused with coding sequences for the $\mathrm{C}_{\kappa}$ and $\mathrm{C}_{\mathrm{H}} 1$ domains (subclass $\mathrm{IgG} 1$ ) of human origin. Although the yields of the humanized rIN-1 Fab fragment were higher than those for the corresponding construct with the murine sequences

(Bandtlow et al., 1996) at the shaker flask scale, the yields were less reproducible in the fermentation, possibly because of an increased toxicity of the recombinant protein for $E$. coli. This problem was solved by introducing a $\mathrm{Cm}$ resistance gene (Fig. $1 A$ ) instead of the previously used ampicillin (Ap) resistance to raise the stringency of antibiotic selection on the expression vector, thus preventing plasmid loss. Yields up to $200 \mathrm{mg}$ of the purified humanized rIN-1 Fab fragment were hence obtained from an 81 fermenter culture. In an attempt to modify antigen binding and functional neutralization, several mutated rIN-1 Fab fragments were prepared. Exchange of single amino acid residues in a region that on the basis of computer modeling was expected to be involved in antigen interaction, resulted in mutants with decreased or completely absent neutralizing activity on Nogo. One of these mutants, with an amino acid exchange from alanine to phenylalanine in position 32, was selected as negative control for the in vivo experiments because of its complete lack of Nogo neutralizing activity (Fig. 3).

\section{rIN-1 Fab stains CNS white matter and recognizes recombinant Nogo-A}

On spinal cord cross sections, prominent staining of myelinated structures of the CNS could be observed (Fig. $2 A$ ). White matter and myelinated fibers in gray matter were strongly stained, whereas the myelin-free dorsal horn exhibited only background staining. Peripheral myelin of the dorsal and ventral roots (Fig. 2A, asterisks) stained only very weakly, reflecting the previously described distribution of the IN-1 antigen in adult nervous tissue. On control 


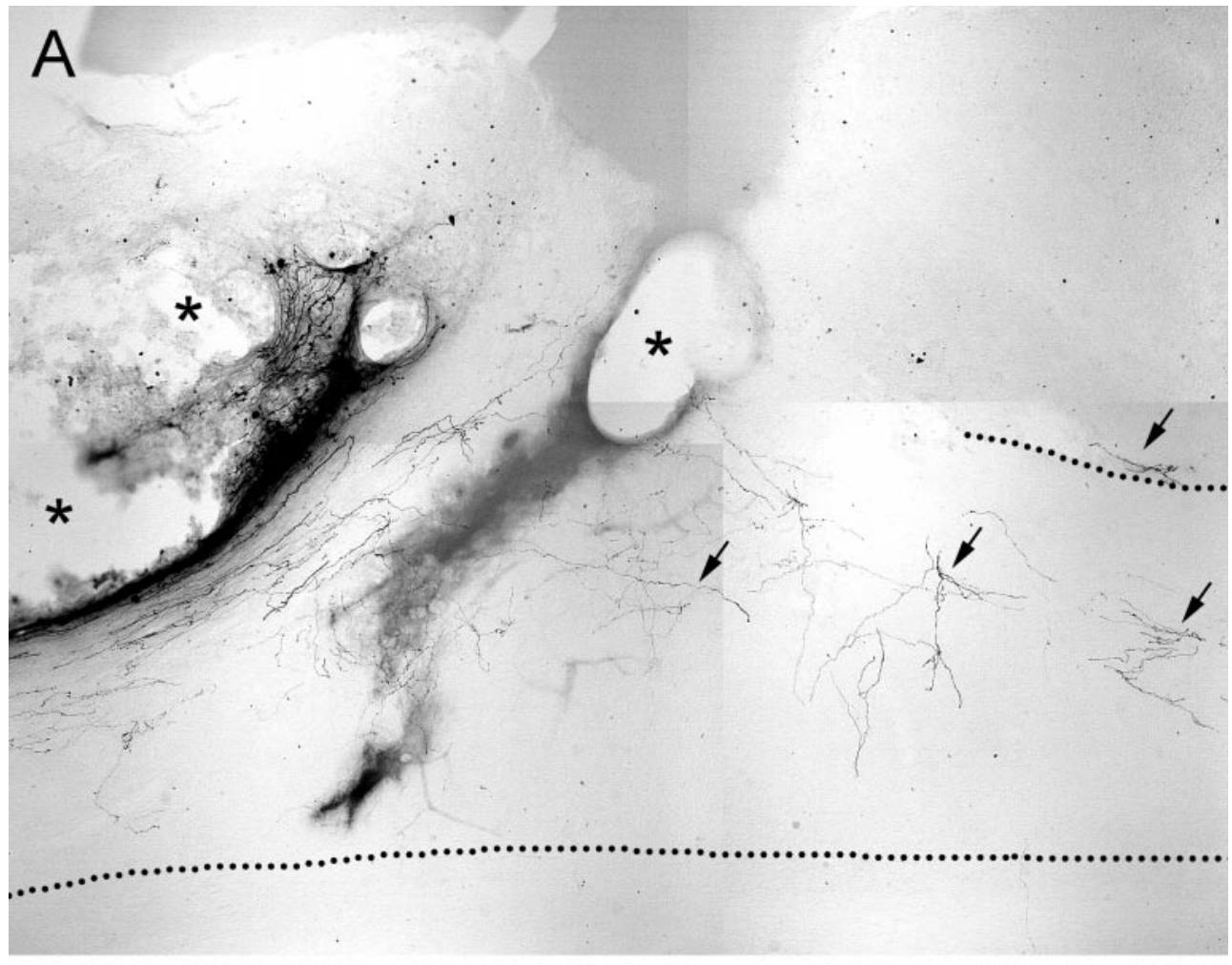

Figure 5. Two examples of BDA-labeled corticospinal fibers at the lesion site in animals treated with rIN-1. Sagittal $50 \mu \mathrm{m}$ vibratome sections. $A$, Overview of a typical lesion area in a rIN-1 Fab-treated animal 2 weeks after injury. The labeled corticospinal tract, which runs at the medioventral aspect of the dorsal funiculus in the rat, approaches the lesion from rostral (left) and is interrupted at the lesion. Cellular infiltrates and cavities are present (asterisks). Arrows indicate regenerated fibers that have circumvented the lesion area and are growing in a typical curved and irregular pattern away from the lesion in the caudal direction. $B$, A close-up in a different rIN-1 animal shows that regenerating fibers growing around and through the lesion are very tortuous and curved. Scale bar (shown in $B): A, 100 \mu \mathrm{m} ; B, 25 \mu \mathrm{m}$.

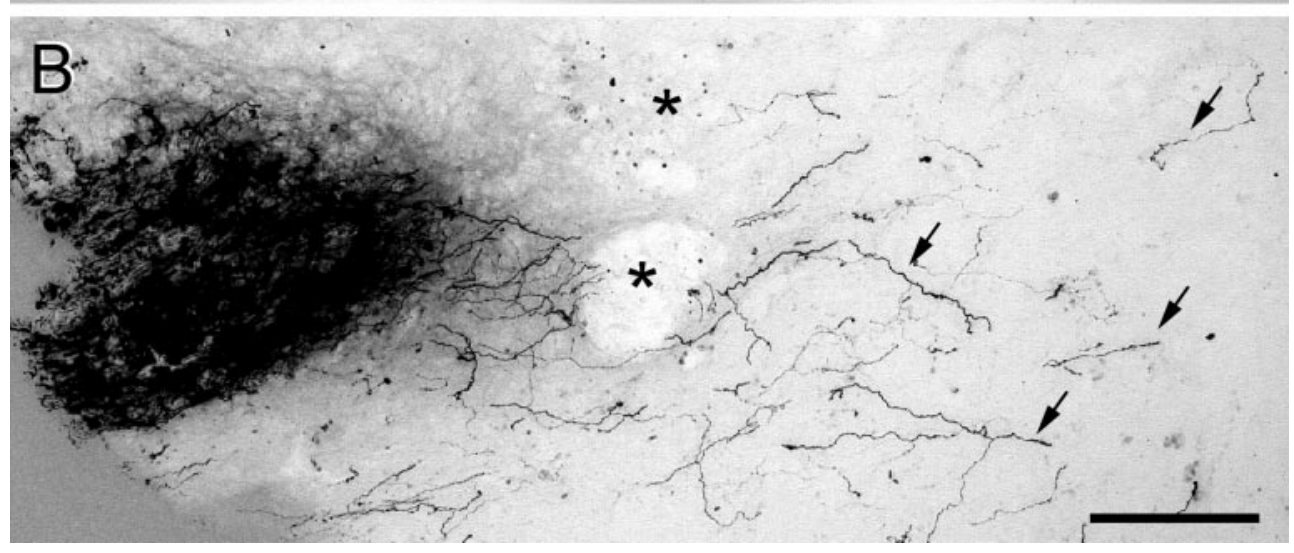

sections (secondary antibody only), no significant staining was observed (Fig. 2B). In conclusion, recombinant humanized IN-1 Fab recognizes the same antigen in CNS tissue as the original mAb IN-1 and its nonderivatized recombinant Fab (Rubin et al., 1994; Bandtlow et al., 1996).

rIN-1 Fab also recognizes recombinant Nogo-A on a Western blot. Full-length myc-tagged Nogo-A prepared from a stably transfected $\mathrm{CHO}$ cell line was run on SDS-PAGE and blotted onto a membrane. rIN-1 Fab was found to recognize the same band as an antibody against the myc-tag of the recombinant protein (Fig. 2C).

\section{rIN-1 Fab neutralizes the myelin-associated inhibitor Nogo-A in vitro}

To determine whether the humanized rIN-1 Fab has retained its inhibitor-neutralizing activity after the exchange of the murine constant domains for human constant domains, two standard in vitro bioassays were used: neurite outgrowth from DRG explants and spreading of 3T3 fibroblasts. Neurite outgrowth and fibroblast spreading are strongly inhibited on culture dishes coated with CNS myelin or a protein extract enriched in Nogo-A (q-pool) (Spillmann et al., 1998). When the inhibitory activity is neutralized by e.g., mAb IN-1, DRG neurite outgrowth and 3T3 spreading are restored to a large extent (Spillmann et al., 1997).

Used at a concentration of 500 and $50 \mu \mathrm{g} / \mathrm{ml}$, the humanized
rIN-1 Fab exhibited strong neutralizing activities in both the 3T3 fibroblast spreading and the DRG neurite outgrowth assays (Fig. 3). 3T3 spreading on the myelin substrate (q-pool) was greatly facilitated $[p<0.01$, Student's $t$ test, compared with q-pool (Spillmann et al., 1998)] to an extent similar to the original mAb IN-1. At higher dilutions of rIN-1 Fab, the neutralizing activity was progressively lost. Mutation of the rIN-1 Fab in position 32 from alanine to phenylalanine (mut rIN-1 Fab) completely abolished the neutralizing activity even at high concentrations (Fig. $3 A$ ).

In the DRG neurite outgrowth assay, humanized rIN-1 Fab at 50 $\mu \mathrm{g} / \mathrm{ml}$ showed similar inhibitor neutralizing activity as the mAb IN-1 hybridoma culture supernatant: DRGs plated on myelin proteins extended no neurites or only very few and short ones. After neutralization of the inhibitory constituents by the humanized rIN-1 Fab, DRGs extended large numbers of long neurites (Fig. $3 B)(p<0.05$, compared with q-pool), an effect that disappeared at higher dilutions of the Fab (data not shown). Also, here mut rIN-1 Fab had lost all neutralizing activity (Fig. $3 B$ ). These results show that a recombinantly expressed, monovalent Fab fragment of the IN-1 antibody has retained the activity of the original decavalent IN-1 IgM and that replacing the murine constant domains with human constant domains did not reduce the inhibitor-neutralizing activity of this rIN-1 Fab. In contrast, rIN-1 Fab carrying a single 
deleterious amino acid exchange $(\mathrm{A} 32 \mathrm{~F})$ in its antigen-binding site did not show any Nogo-A-neutralizing activity.

\section{Histology of lesion and rIN-1 Fab distribution}

Rats were subjected to a dorsal spinal cord hemisection at level T8, a tracer injection into the sensorimotor cortex, and received continuous infusions close to the lesion site of either rIN-1 Fab, mut rIN-1 Fab, or BSA (Fig. 4A). After a survival time of 2 weeks, animals were killed, and the spinal cord was processed for the visualization of the CST. At that time, secondary processes had considerably enlarged the initial small and well defined lesion site. Scars and cavities had formed, and large numbers of cells (mostly macrophages and neutrophils) had infiltrated the area (Fig. 5A,B). Thus, these lesions closely resembled lesions with no further treatment or lesions obtained by contusion techniques (Beattie et al., 1997). In some animals the infusion catheter had caused a mild compression of the caudal cord with concomitant cell infiltrations. To study the tissue distribution of rIN-1 Fab, the recombinant antibody was coupled to the fluorescent dye Alexa488. As revealed by fluorescence microscopy, after 1 week of inf usion, Alexa488rIN-1 Fab had distributed within at least $20 \mathrm{~mm}$ rostral and caudal of the infusion site in all animals $(n=3)$. Alexa488 fluorescence appeared somewhat higher in gray matter areas but was also clearly visible in both dorsal and ventral white matter tracts (Fig. $4 B$ ). Thus, the rIN-1 $\mathrm{Fab}$ is clearly present in regions where regenerative fiber growth was observed.

\section{rIN-1 Fab induces regeneration of transected spinal cord axons in adult rats}

Rostral to the lesion the transected corticospinal axons had retracted somewhat from the site of injury. In both the experimental as well as the control groups, many sprouts extended from the cut axons into adjacent tissue. In the control groups (infused with BSA or mutated, inactive rIN-1 Fab), these sprouts did not grow beyond the middle of the lesion area (Figs. $6 B, 8 E$ ). In contrast, in 11 of 18 animals treated with $\mathrm{rIN}-1 \mathrm{Fab}$, fibers emerging from the proximal CST stump bypassed the lesion area and grew toward the caudal end of the spinal cord (Figs. 5, 6A, $8 A-D$ ). Most of these regenerating axons grew through remaining tissue bridges of ventral and ventrolateral gray and white matter (Fig. 5, 6A). In the vicinity of the lesion, the course of these fibers was typically tortuous and very distinct from the normal straight fiber morphology in the CST. In the caudal spinal cord the regenerating fibers often grew in a more straight fashion, especially within tract areas. Collaterals in the gray matter were frequent, and these arborized often very extensively (Fig. 7). These terminal arborizations were decorated with many varicosities (Fig. $7 C-F$ ) that strongly resembled presynaptic boutons on normal CST arborizations rostral to the lesion (Fig. $7 G$ ).

Reconstructions of consecutive sections as camera lucida drawings revealed the overall pattern of CST fiber growth (Figs. 6, 8). Regenerating CST fibers seemed often to prefer the dorsal half of the spinal cord, including the former CST territory, but also grew in other regions. Extensive ramifications into gray matter areas were observed at all levels. From the camera lucida reconstructions, the maximal regeneration distance of each animal was determined as the distance from the center of the lesion to the most caudally extending regenerated axons. Maximal regeneration distances varied from rat to rat and reached values from 1.4 to $>9 \mathrm{~mm}$ (Fig. 9). Absence of regenerating CST axons in rIN-1 Fab-treated animals correlated in some (e.g., Fig. $8 C$ ) but not all animals with very large lesions and extensive formation of scars and cavities.

\section{DISCUSSION}

A recombinant, humanized Fab fragment of the mAb IN-1 was produced in bacteria and shown to be able to efficiently neutralize the neurite growth inhibitory properties of a CNS myelin preparation enriched for the neurite growth inhibitor Nogo-A in vitro. In addition, it stains myelinated CNS tissue very similarly to the original IN-1 mAb and its nonhumanized recombinant Fab and recognizes recombinant Nogo-A on a Western blot. We therefore
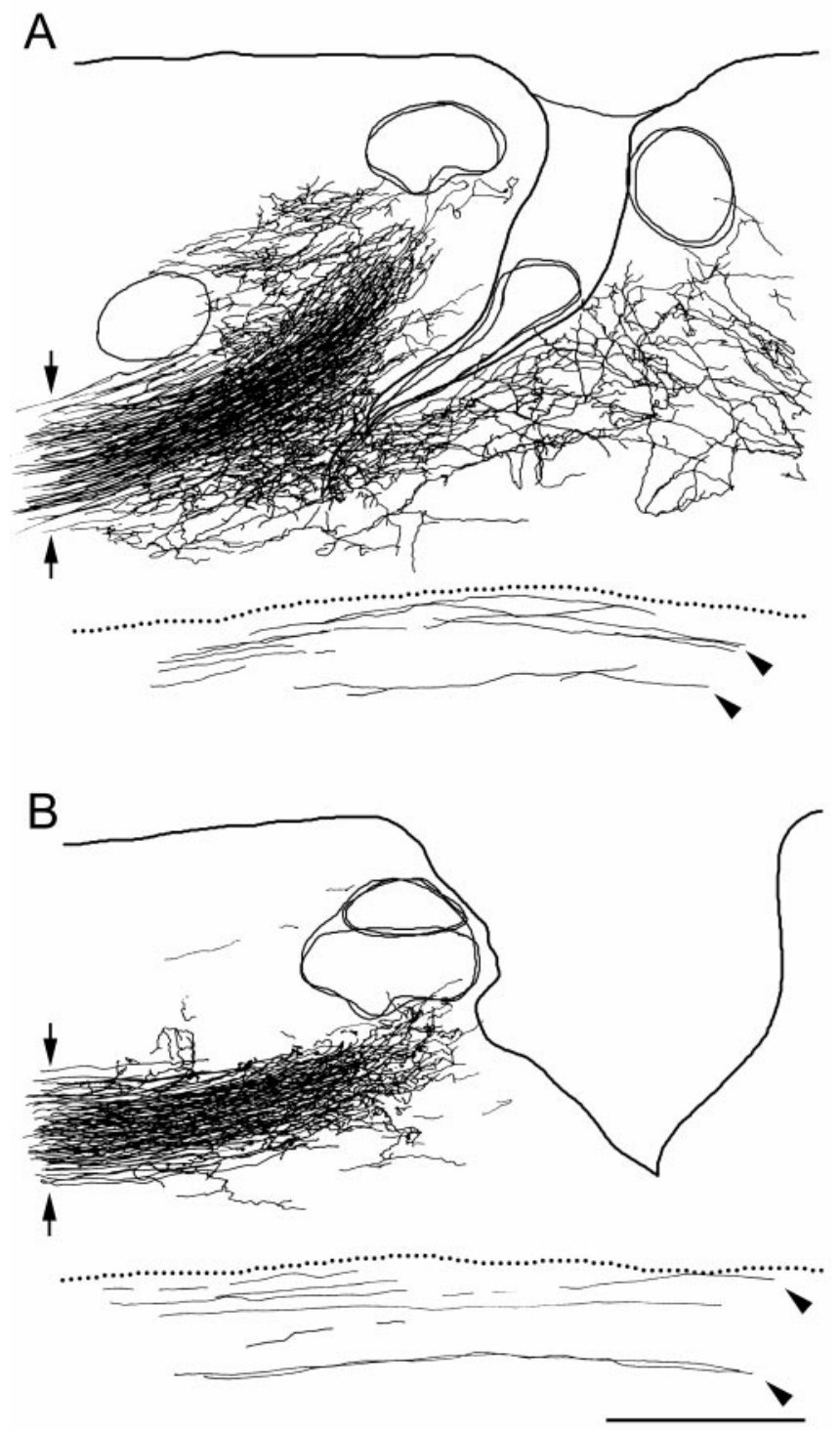

Figure 6. Camera lucida reconstructions of consecutive sections fibers at the lesion site in an animal infused with $\mathrm{rIN}-1 \mathrm{Fab}(A)$ and a control animal infused with mut rIN-1 Fab $(B)$. In the rIN-1 Fab-infused animal, strong sprouting of the lesioned CST fibers can be observed. Some of these sprouts elongate and grow around and through the lesion and farther down the cord. In the mut rIN-1-infused animal, some sprouts have emanated from the lesioned CST, but no long-distance growth occurs. The number of labeled CST fibers and their labeling intensity (arrows), lesion depth, and size are similar in the two animals. Scale bar, $500 \mu \mathrm{m}$.

conclude that humanized rIN-1 Fab has fully retained the specificity and activity of the original IN-1 mAb. Currently, experiments are under way to further characterize the exact epitope where the rIN-1 Fab binds to Nogo-A.

When infused subdurally at the site of a partial spinal cord transection in adult rats, rIN-1 promoted long-distance axonal re-growth within 2 weeks. Regenerating corticospinal tract fibers were reconstructed in camera lucida drawings of consecutive sections, and only fibers that could be traced back to the completely lesioned, main dorsomedial CST component were analyzed further. Care was taken not to confuse these fibers with unlesioned, ipsilateral, ventral CST fibers. Regenerated axons branched into gray matter areas where their arborizations were decorated with putative synaptic boutons. No growth beyond normal lesioninduced sprouting was observed in animals treated with a mutated, inactive rIN-1 Fab or BSA solution as controls. In the rIN-1 Fab experimental group, regenerated fibers extended between 1.4 and $9.6 \mathrm{~mm}$ beyond the site of injury within 2 weeks after injury, with 


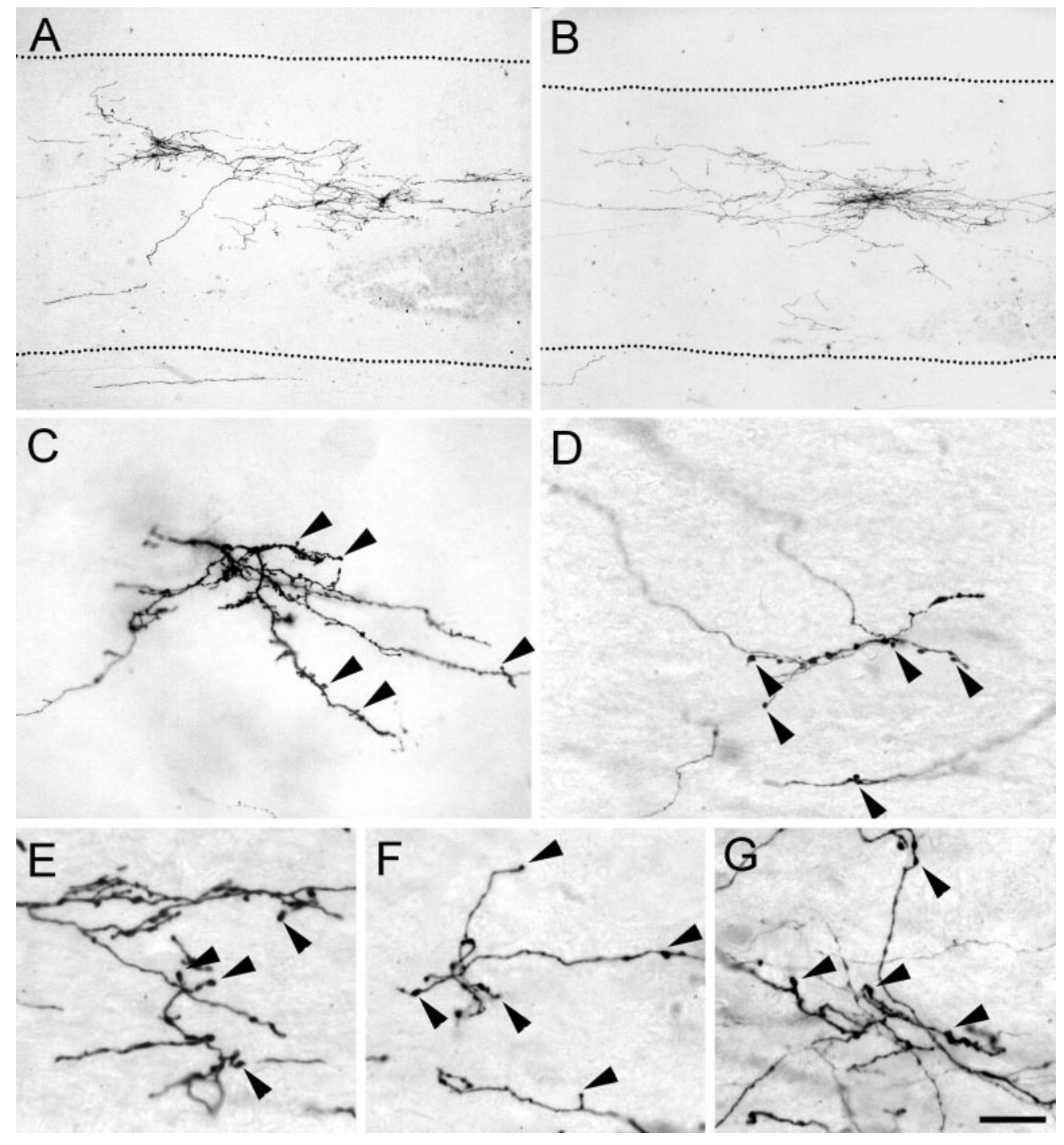

Figure 7. Regenerated corticospinal fibers in the spinal cord distal to the lesion site branch prof usely and give rise to large arborizations in intermediate laminae of the cord $(A, B)$. Higher magnification $(C-F)$ reveals that these arborizations are decorated with many varicosities (arrowheads) that strongly resemble presynaptic boutons in normal corticospinal innervation of spinal gray matter $(G)$. Both terminal and en passant presynaptic boutons can be identified. Parasagittal sections are $50 \mu \mathrm{m}$ thick. Scale bar (shown in $G$ ): $A, B$, $250 \mu \mathrm{m} ; C, D, 30 \mu \mathrm{m} ; E-G, 15 \mu \mathrm{m}$.

many of them reaching lumbar spinal levels. Interestingly, the extent and distance of regenerating fibers were similar to that reported previously for mAb IN-1 induced regeneration (Schnell and Schwab, 1993; Schnell et al., 1994), although the procedure of antibody application by an osmotic minipump directly to the lesion site allowed much better control over the amount and distribution of infused antibody. Although the antibody concentration at the site of injury is likely to be much higher in this study, the lower avidity of the only monovalent rIN-1 Fab when compared with the decavalent mAb IN-1 (Bandtlow et al., 1996) may lead to a weaker growth-promoting effect. As in earlier experiments using hybridoma suspension grafts or encapsulated hybridoma implants (Schnell and Schwab, 1993; Schnell et al., 1994), the interindividual variation in the rIN-1 Fab treatment group was large and 7 of 18 animals did not respond to the treatment. We also observed that lesions with large rostrocaudal extent correlated with poor regenerative growth in some animals. This may reflect increased growth-adverse effects of the scar, the local inflammatory reaction, the larger blood-brain barrier breakdown area (Jaeger and Blight, 1997), and other inhibitory molecules such as proteoglycans (McKeon et al., 1991; Davies et al., 1997; Fitch and Silver, 1997), ephrins (Miranda et al., 1999), or semaphorins (Pasterkamp et al., 1998, 1999) that are known to be associated with scars and to be upregulated after a CNS lesion and inflammation. Whether Nogo-A expression increases at the lesion site in response to injury is not known and is currently under investigation in our laboratory.

Anterograde tracing of CST axons by BDA allowed exact determination of the trajectories of regenerating fibers. Although most
CST fibers normally grow in the dorsal funiculus, regenerating fibers were found to sprout from the bulk of transected fibers rostral from the lesion site and bypass the injury through remaining ventral gray and white matter. The growth at this level was typically very curved and tortuous, probably because of the compromised tissue integrity in the vicinity of the lesion. When the regenerated fibers had passed the lesion, their trajectories became straighter, and ramifications and arborizations in gray matter areas could be observed. Arborizations in gray matter areas were decorated with numerous bouton-like swellings that very much resembled the presynaptic boutons on normal CST collaterals innervating the spinal gray matter. We therefore suggest that regenerated fibers may have formed again synaptic contacts with neurons in the spinal cord distal to the injury.

Tortuous growth and extensive ramification and collateralization prohibited exact quantitative analysis of fiber numbers. However, analysis of camera lucida drawings of consecutive sections demonstrated that the regenerating fibers arborized profusely into large gray matter areas caudal to the lesion. We have shown earlier (Brösamle and Schwab, 1997) that the tracing method used in this study labels only a fraction of $\sim 1-2 \%$ of all corticospinal fibers. Therefore, the actual number of regenerated fibers is likely to be considerably higher than what was observed in our experiments. This regenerative regrowth together with plastic fiber growth of unlesioned systems (Thallmair et al., 1998; Z'Graggen et al., 1998) may, through reinnervation of spinal neurons, account for the strong recovery-enhancing effects of IN-1 treatment observed earlier (Bregman et al., 1995; Thallmair et al., 1998; Z'Graggen et al., 

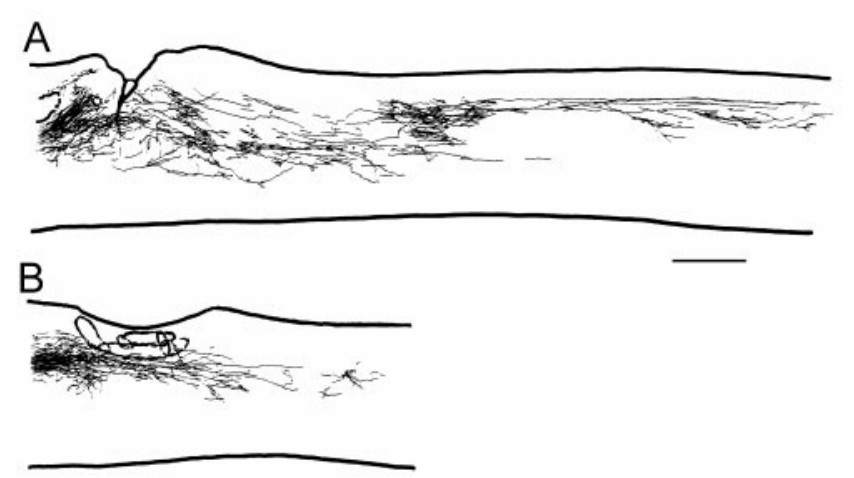

C
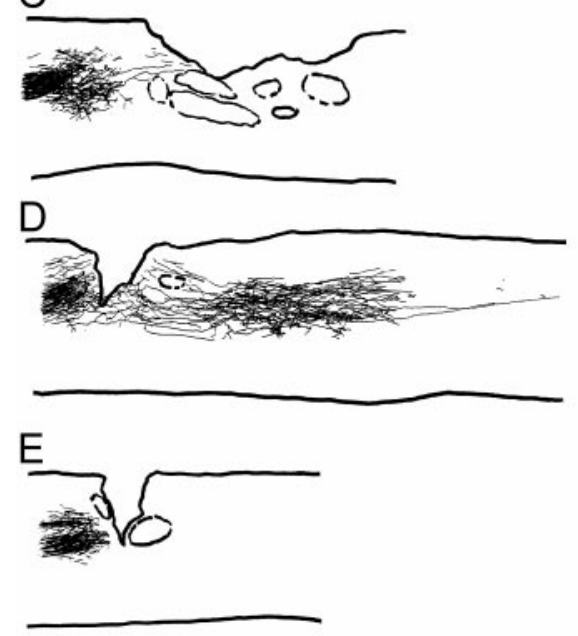

Figure 8. Camera lucida reconstructions of consecutive series of parasagittal sections $(50 \mu \mathrm{m}$ thick) of the thoracic and high lumbar spinal cord of four representative rIN-1 Fab-treated animals $(A-D)$ and a representative control animal $(E)$. Most rIN-1 Fab-treated animals exhibited longdistance regeneration for up to $>9 \mathrm{~mm}$ with axons branching prof usely into gray matter areas, whereas control animals showed no regenerative growth. Scale bar, $1 \mathrm{~mm}$.

1998). An important role may be played by the lumbar spinal locomotor pattern networks, which have been shown to be preserved in animals with spinal lesions above the T12 segment (Grillner, 1975; Edgerton et al., 1992). Interestingly, most human spinal cord injury patients, including many with neurologically complete lesions, have remaining bridges of spinal cord tissue at the lesion site; persisting locomotor pattern generators could also be demonstrated in human paraplegic patients (Dietz et al., 1994).

From an analysis of the cloned sequences of the light chain variable domain, it can be deduced that the original $\mathrm{mAb} \mathrm{IN}-1$ is derived from an early immune response and has not undergone somatic hypermutation with affinity maturation (Bandtlow et al., 1996). Its binding affinity therefore may be relatively low. Experiments are currently underway not only to humanize the variable regions of the Fab fragment but also to improve antigen recognition and neutralization by further engineering the antigen binding site. So far this has led to a mutated IN-1 Fab that has lost its neutralizing activity and was used as a control in the experiments reported in this study. Increased antigen affinity of a third generation rIN-1 Fab fragment might lead, however, to enhanced regenerationinducing capabilities and improved functional recovery after injury.

With the humanized rIN-1 Fab, a tool is now available that can permit injured CNS nerve fibers to regenerate over long distances by neutralizing myelin-associated growth inhibitory proteins in spinal cord and brain. This represents an important step toward a potential treatment because the rIN-1 Fab could be applied in a way that is routinely used for application of substances like ba-

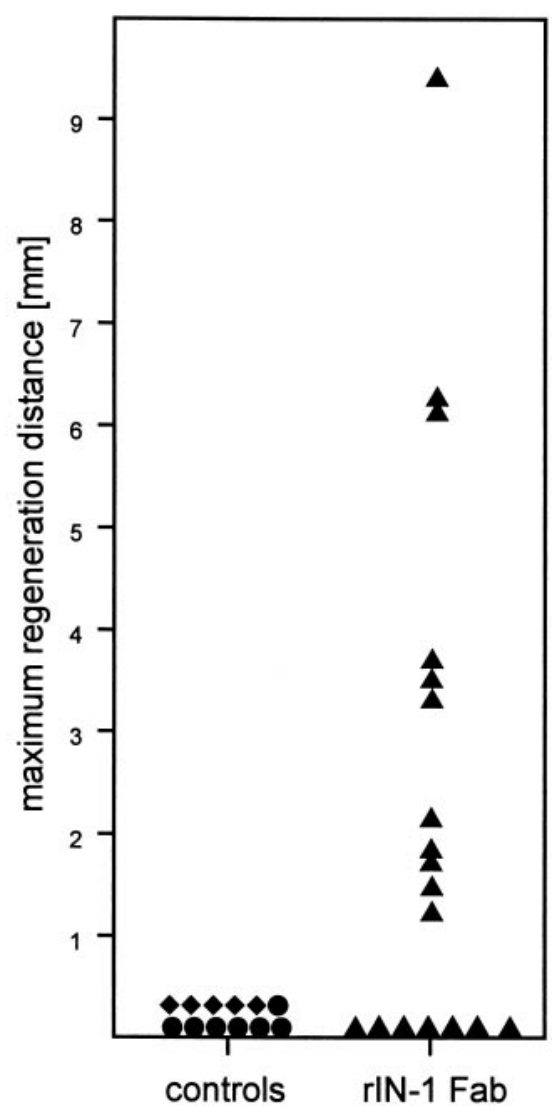

Figure 9. Maximal elongation of regenerating axons in response to control $(\mathbf{O}, \mathrm{BSA} ; \boldsymbol{\bullet}$, mut rIN-1 Fab) or rIN-1 Fab $(\boldsymbol{\Delta})$ treatment. Every symbol represents one animal and indicates the distance of the longest regenerated CST fiber from the center of the lesion. Although in the rIN-1 Fab group 11 of 18 animals showed considerable regrowth of transected CST fibers, this was never observed in control animals.

clofen to the spinal cord in human patients (Penn et al., 1989; Lazorthes et al., 1990).

\section{REFERENCES}

Bandtlow C, Schiweck W, Tai HH, Schwab ME, Skerra A (1996) The Escherichia coli-derived Fab fragment of the IgM/kappa antibody IN-1 recognizes and neutralizes myelin-associated inhibitors of neurite growth. Eur J Biochem 241:468-475.

Beattie MS, Bresnahan JC, Komon J, Tovar CA, Van Meter M, Anderson DK, Faden AI, Hsu CY, Noble LJ, Salzman S, Young W (1997) Endogenous repair after spinal cord contusion injuries in the rat. Exp Neurol 148:453-463.

Bregman BS, Kunkel-Bagden E, Schnell L, Dai HN, Gao D, Schwab ME (1995) Recovery from spinal cord injury mediated by antibodies to neurite growth inhibitors. Nature 378:498-501.

Brösamle C, Schwab ME (1997) Cells of origin, course, and termination patterns of the ventral, uncrossed component of the mature rat corticospinal tract. J Comp Neurol 386:293-303.

Cadelli D, Schwab ME (1991) Regeneration of lesioned septohippocampal acetylcholinesterase-positive axons is improved by antibodies against the myelin-associated neurite growth inhibitors NI-35/250. Eur J Neurosci 3:825-832.

Caroni P, Schwab ME (1988) Antibody against myelin-associated inhibitor of neurite growth neutralizes nonpermissive substrate properties of CNS white matter. Neuron 1:85-96.

Chen MS, Huber AB, van der Haar ME, Frank M, Schnell L, Spillmann AA, Christ F, Schwab ME (2000) Nogo-A is a myelin-associated neurite outgrowth inhibitor and an antigen for monoclonal antibody IN-1. Nature 403:434-439.

Davies SJ, Fitch MT, Memberg SP, Hall AK, Raisman G, Silver J (1997) Regeneration of adult axons in white matter tracts of the central nervous system. Nature 390:680-683.

Dietz V, Colombo G, Jensen L (1994) Locomotor activity in spinal man. Lancet 344:1260-1263.

Edgerton VR, Roy RR, Hodgson JA, Prober RJ, de Guzman CP, de Leon $R$ (1992) Potential of adult mammalian lumbosacral spinal cord to exe- 
cute and acquire improved locomotion in the absence of supraspinal input. J Neurotrauma 9:119-128.

Fiedler M, Skerra A (1999) Use of thiophilic adsorption chromatography for the one-step purification of a bacterially produced antibody $\mathrm{Fab}$ fragment without the need for an affinity tag. Protein Expr Purif 17:421-427.

Fitch MT, Silver J (1997) Activated macrophages and the blood-brain barrier: inflammation after CNS injury leads to increases in putative inhibitory molecules. Exp Neurol 148:587-603.

GrandPré T, Nakamura F, Vartanian T, Strittmatter SM (2000) Identification of the Nogo inhibitor of axon regeneration as a reticulon protein. Nature 403:439-444.

Grillner S (1975) Locomotion in vertebrates: central mechanisms and reflex interaction. Physiol Rev 55:247-304.

Herzog A, Brösamle C (1997) "Semifree-floating" treatment: a simple and fast method to process consecutive sections for immunohistochemistry and neuronal tracing. J Neurosci Methods 72:57-63.

Jaeger CB, Blight AR (1997) Spinal cord compression injury in guinea pigs: structural changes of endothelium and its perivascular cell associations after blood-brain barrier breakdown and repair. Exp Neurol 144:381-399.

Lazorthes Y, Sallerin-Caute B, Verdie JC, Bastide R, Carillo JP (1990) Chronic intrathecal baclofen administration for control of severe spasticity. J Neurosurg 72:393-402.

McKeon RJ, Schreiber RC, Rudge JS, Silver J (1991) Reduction of neurite outgrowth in a model of glial scarring following CNS injury is correlated with the expression of inhibitory molecules on reactive astrocytes. J Neurosci 11:3398-3411.

Miranda JD, White LA, Marcillo AE, Willson CA, Jagid J, Whittemore SR (1999) Induction of Eph B3 after spinal cord injury. Exp Neurol $156: 218-222$.

Orlandi R, Gussow DH, Jones PT, Winter G (1989) Cloning immunoglobulin variable domains for expression by the polymerase chain reaction. Proc Natl Acad Sci USA 86:3833-3837.

Pasterkamp RJ, De Winter F, Holtmaat AJ, Verhaagen J (1998) Evidence for a role of the chemorepellent semaphorin III and its receptor neuropilin-1 in the regeneration of primary olfactory axons. J Neurosci 18:9962-9976.

Pasterkamp RJ, Giger RJ, Ruitenberg MJ, Holtmaat AJ, De Wit J, De Winter F, Verhaagen J (1999) Expression of the gene encoding the chemorepellent semaphorin III is induced in the fibroblast component of neural scar tissue formed following injuries of adult but not neonatal CNS. Mol Cell Neurosci 13:143-166.

Penn RD, Savoy SM, Corcos D, Latash M, Gottlieb G, Parke B, Kroin JS (1989) Intrathecal baclofen for severe spinal spasticity. N Engl J Med 320:1517-1521.

Plückthun A, Skerra A (1989) Expression of functional antibody Fv and Fab fragments in Escherichia coli. Methods Enzymol 178:497-515.

Ramon y Cajal S (1928) Degeneration and regeneration of the nervous system. New York: Hafner.

Rubin BP, Dusart I, Schwab ME (1994) A monoclonal antibody (IN-1) which neutralizes neurite growth inhibitory proteins in the rat CNS recognizes antigens localized in CNS myelin. J Neurocytol 23:209-217.

Rubin BP, Spillmann AA, Bandtlow CE, Keller F, Schwab ME (1995) Inhibition of PC12 cell attachment and neurite outgrowth by detergent solubilized CNS myelin proteins. Eur J Neurosci 7:2524-2529.

Schiweck W, Skerra A (1995) Fermenter production of an artificial fab fragment, rationally designed for the antigen cystatin, and its optimized crystallization through constant domain shuffling. Proteins 23:561-565.

Schiweck W, Buxbaum B, Schatzlein C, Neiss HG, Skerra A (1997) Sequence analysis and bacterial production of the anti-c-myc antibody 9E10: the V(H) domain has an extended CDR-H3 and exhibits unusual solubility. FEBS Lett 414:33-38.

Schnell L, Schwab ME (1990) Axonal regeneration in the rat spinal cord produced by an antibody against myelin-associated neurite growth inhibitors. Nature 343:269-272.

Schnell L, Schwab ME (1993) Sprouting and regeneration of lesioned corticospinal tract fibres in the adult rat spinal cord. Eur J Neurosci 5:1156-1171.

Schnell L, Schneider R, Kolbeck R, Barde Y-A, Schwab ME (1994) Neurotrophin-3 enhances sprouting of corticospinal tract during development and after adult spinal cord lesion. Nature 367:170-173.

Schwab ME, Bartholdi D (1996) Degeneration and regeneration of axons in the lesioned spinal cord. Physiol Rev 76:319-370.

Schwab ME, Caroni P (1988) Oligodendrocytes and CNS myelin are nonpermissive substrates for neurite growth and fibroblast spreading in vitro. J Neurosci 8:2381-2393.

Schwab ME, Thoenen H (1985) Dissociated neurons regenerate into sciatic but not optic nerve explants in culture irrespective of neurotrophic factors. J Neurosci 5:2415-2423.

Schwab ME, Kapfhammer JP, Bandtlow CE (1993) Inhibitors of neurite growth. Annu Rev Neurosci 16:565-595.

Skerra A, Plückthun A (1988) Assembly of a functional immunoglobulin Fv fragment in Escherichia coli. Science 240:1038-1041.

Spillmann AA, Amberger VR, Schwab ME (1997). High molecular weight protein of human central nervous system myelin inhibits neurite outgrowth: an effect which can be neutralized by the monoclonal antibody IN-1. Eur J Neurosci 9:549-555.

Spillmann AA, Bandtlow CE, Lottspeich F, Keller F, Schwab ME (1998) Identification and characterization of a bovine neurite growth inhibitor (bN I-220). J Biol Chem 273:19283-19293.

Thallmair M, Metz GAS, Z'Graggen WJ, Raineteau O, Kartje GL, Schwab ME (1998) Neurite growth inhibitors restrict structural plasticity and functional recovery following corticospinal tract lesions. Nat Neurosci $1: 124-131$.

Weibel D, Cadelli D, Schwab ME (1994) Regeneration of lesioned rat optic nerve fibers is improved after neutralization of myelin-associated neurite growth inhibitors. Brain Res 642:259-266.

Z'Graggen W, Metz GAS, Kartje GL, Thallmair M, Schwab ME (1998) Functional recovery and enhanced corticofugal plasticity after unilateral pyramidal tract lesion and blockade of myelin-associated neurite growth inhibitors in adult rats. J Neurosci 18:4744-4757. 